NASA TECHNICAL NOTE

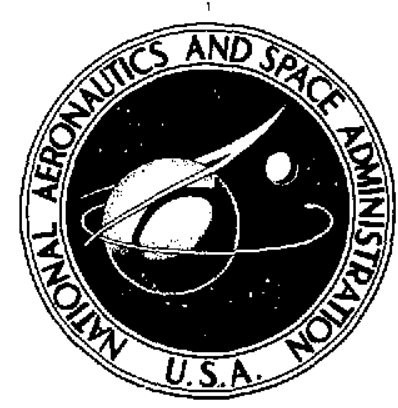

NASA TN D-7828

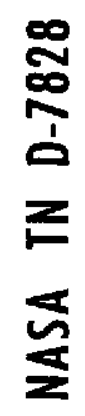



\title{
ANALYSIS OF VALIDATION TESTS OF THE LANGLEY PILOT TRANSONIC CRYOGENIC TUNNEL
}

Edward J. Ray, Robert A. Kilgore, Jerry $B$. and Edwin E. Davenport

Langley Research Center

Hampton, Va. 23665

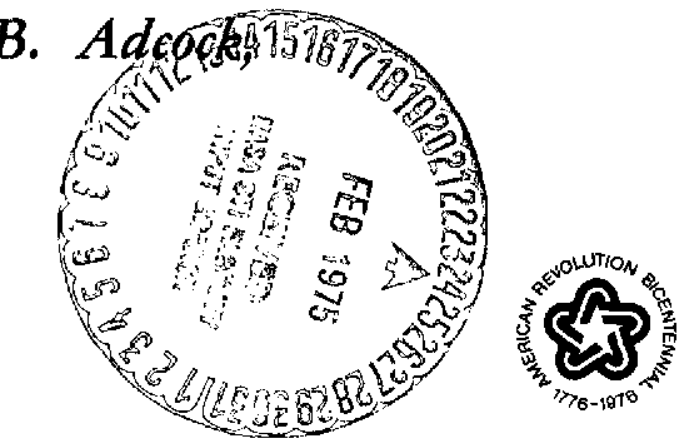

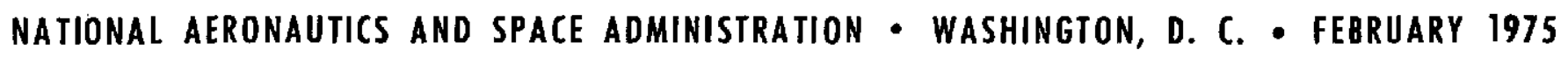




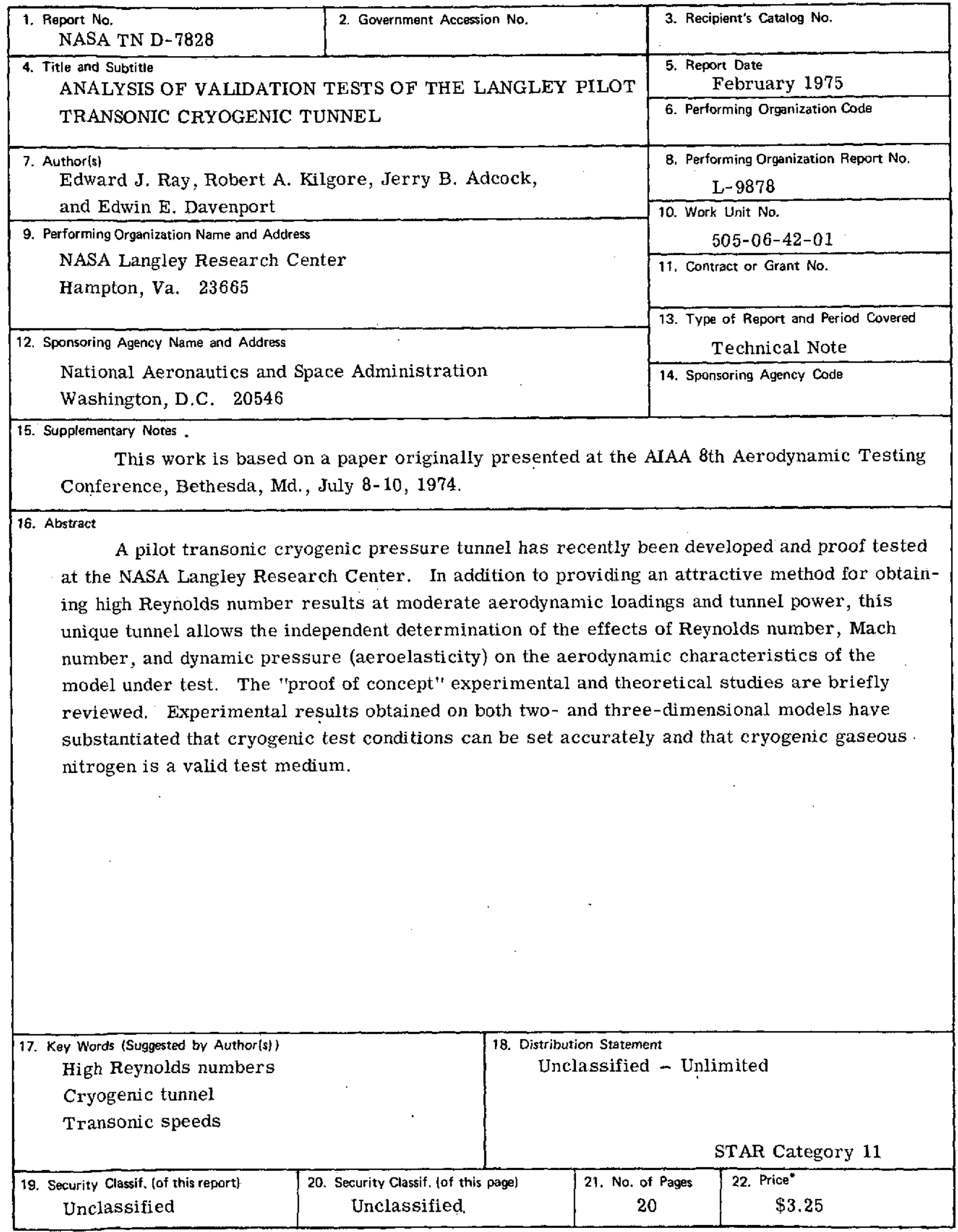

"For sale by the National Technical Information Service, Springfield, Virginia 22151 


\section{ANALYSIS OF VALIDATION TESTS OF THE LANGLEY PILOT TRANSONIC CRYOGENIC TUNNEL*

\author{
Edward J. Ray, Robert A. Kilgore, Jerry B. Adcock, \\ and Edwin E. Davenport \\ Langley Research Center
}

\section{SUMMARY}

A pilot transonic cryogenic pressure tunnel has recently been developed and proof tested at the NASA Langley Research Center. In addition to providing an attractive method for obtaining high Reynolds number results at moderate aerodynamic loadings and tunnel power, this unique tunnel allows the independent determination of the effects of Reynolds number, Mach number, and dynamic pressure (aeroelasticity) on the aerodynamic characteristics of the model under test. The "proof of concept" experimental and theoretical studies are briefly reviewed. Experimental results obtained on both two- and three-dimensional models have substantiated that cryogenic test conditions can be set accurately and that cryogenic gaseous nitrogen is a valid test medium.

\section{INTRODUCTION}

For many years the wind tunnel has proved to be a valuable experimental device which has played an important role in the improvement of aircraft performance and flying qualities and facilitated the development of advanced aerodynamic concepts. The development of new wind tunnels has kept pace with the advances in flight Mach number capability. However, the need for substantial increases in the Reynolds number capability has become apparent in recent years, particularly at high subsonic and transonic speeds, where shock-boundary-layer interactions and other viscous effects can have an unusually large influence on stability and performance characteristics. In this regard, it is generally accepted that the capability should exist for minimum test Reynolds numbers, based on the mean aerodynamic chord, on the order of $50 \times 10^{6}$. The above goal represents an increase in Reynolds number capability by about a factor of 5 over that of existing transonic tunnels in the United States.

Although the necessity for transonic wind tunnels capable of achieving high Reynolds numbers has been recognized by researchers for many years, the problems imposed by

*Based on a paper originally presented at the AIAA 8th Aerodynamic Testing Conference, Bethesda, Md., July 8-10, 1974. 
economics and lack of technology have until recently made it impractical to construct a facility of this type. Nevertheless, design approaches to achieve high test Reynolds numbers have been the subject of intense studies for a number of years. One of the most innovative concepts to surface was to operate wind tunnels at cryogenic temperatures. This concept was first proposed by Smelt in 1945 (ref. 1) as an attractive method for achieving high test Reynolds numbers at reasonable levels of drive power in fan-driven tunnels. However, at the time of Smelt's work, the lack of a practical means of cooling a wind tunnel to cryogenic temperatures and the unavailability of suitablesstructural materials precluded the application of the cryogenic wind-tunnel concept." During the latter part of 1971, a study was begun at the Langley Research Center to determine the feasibility of applying the cryogenic concept to a high Reynolds number transonic wind tunnel. The analyses of Smelt were extended and a small low-speed model tunnel was modified for cryogenic operation (ref. 2). Results obtained from these initial studies stimulated the design and construction of the Langley pilot transonic cryogenic tunnel. This tunnel was placed in operation in September of 1973, and some of the initial results obtained were reported in reference 3. As a result of the successful operation during the validation studies, the pilot cryogenic tunnel has been reclassified by NASA as a research facility and as such is being used for aerodynamic research as well as cryogenic technology studies.

The purpose of this paper is to provide a brief review of the cryogenic concept and to present an analysis of some of the recent experimental results obtained in the Langley pilot cryogenic tunnel.

\section{SYMBOLS}

Values are given both in SI and U.S. Customary Units. Measurements and calculations were made in U.S. Customary Units.

A aspect ratio

c chord of airfoil

$\bar{c} \quad$ mean geometric chord, $13.33 \mathrm{~cm}$ (5.246 in.)

$\mathrm{C}_{\mathrm{N}} \quad$ normal-force coefficient

$C_{p} \quad$ pressure coefficient, $\frac{p-p_{\infty}}{q_{\infty}}$ 
sound pressure level

Mach number

$\mathbf{M}_{l} \quad$ local Mach number

$\Delta \mathrm{M}_{l} \quad$ change in local Mach number

p pressure

$\mathrm{p}_{\mathrm{t}} \quad$ stagnation pressure

$\mathrm{q} \quad$ dynamic pressure, $\frac{1}{2} \rho \mathrm{V}^{2}$

R Reynolds number

$\mathbf{R}_{\mathrm{c}} \quad$ Reynolds number based on chord

$\mathbf{R}_{\overline{\mathrm{c}}} \quad$ Reynolds number based on $\overline{\mathrm{c}}$

T temperature

$\mathrm{T}_{\mathrm{t}} \quad$ stagnation temperature

v local velocity

V free-stream velocity

x linear dimension along airfoil chord line

vertical position above tunnel floor

$\alpha$

angle of attack 


$$
\begin{array}{ll}
\mu & \text { free-stream viscosity } \\
\rho & \text { free-stream density } \\
\sigma & \text { standard deviation }
\end{array}
$$

Subscripts:

$$
\begin{array}{ll}
\infty & \text { free stream } \\
\max & \text { maximum }
\end{array}
$$

Abbreviations:

C.R. center of rotation

2-D two-dimensional model

3-D three-dimensional model

\section{RESULTS AND DISCUSSION}

\section{Why Cryogenics?}

There are several approaches which will provide the desired increase in Reynolds number capability. Reynolds number, which of course is the ratio of the inertia forces to the viscous forces, is given by

$$
\mathrm{R}=\frac{\rho \mathrm{V}^{2} \ell^{2}}{\mu \mathrm{V} \ell}
$$

which reduces to the well-known equations

$$
\mathrm{R}=\frac{\rho \mathrm{V} \ell}{\mu}
$$

or

$$
\mathrm{R}=\frac{\rho \mathrm{Ma} \ell}{\mu}
$$


From the equation for Reynolds number, an obvious way to increase Reynolds number is to increase the model size, that is, to increase $\ell$. For constant wall interference this method requires a commensurate increase in wind-tunnel size, which results in excessively large, costly facilities with prohibitive power requirements. An alternate solution is to restrict the tunnel and model sizes and increase the operating pressure and, consequently, the density $p$. This method is feasible, of course, but the aerodynamic forces on the model, balance, and support system are greatly increased at the operating pressures that would be required to achieve the desired Reynolds number. From a power standpoint, a high-pressure tunnel is preferable to a large, moderate-pressure tunnel. However, for the required increase in Reynolds number, the power requirements are still undesirably large. A third method for increasing Reynolds number is to decrease the temperature of the test gas. As temperature is reduced, the density $\rho$ increases, the viscosity $\mu$ decreases, and the speed of sound a decreases. While the benefits of these effects of cryogenic operation are readily apparent from the usual forms of the Reynolds number equation (eq. (1b) or (1c)), it is informative to inspect the inertia and viscous forces separately in equation (1a). It thus can be shown that the increase in density and decrease in velocity (for a given $\mathbf{M}$ ) cancel, so that there is no change in the inertia force, and a large decrease in the viscous force due both to the decreasing viscosity and decreasing velocity occurs. The result is a large increase in Reynolds number without the large increase in inertia or dynamic pressure that accompanies the pressure-tunnel approach. In addition, the reduced velocity results in large reductions in drive-power requirements and energy consumption (ref. 3 ).

Figure 1 provides some insight into the magnitude of the advantages of testing at cryogenic temperatures. The ratios of several key test parameters to their values at a typical ambient temperature condition are plotted against stagnation temperature. It can



Tigure 1.- Some effects of cryogenic operation. $M_{\infty}=1.0 ; p_{t}=1$ atm. 
readily be seen that at cryogenic temperatures the Reynolds number is increased by a factor of about 6 with no increase in dynamic pressure and with a reduction of about onehalf in the required drive power. There are, in addition, some important advantages with respect to the kinds of testing possible in a tunnel capable of cryogenic operation. As noted in reference 3 , with the unique ability to control tunnel temperature, pressure, and Mach number separately, it is possible to determine independently the effects of Reynolds number, dynamic pressure, and compressibility on the aerodynamic characteristics of the model under test. Because of the many inherent advantages associated with testing at low temperatures, the cryogenic approach appears to be the most desirable method for acquiring transonic test results at high Reynolds number.

\section{Langley Pilot Transonic Cryogenic Tunnel}

The Langley pilot transonic cryogenic tunnel is a continuous flow, fan-driven tunnel with a slotted octagonal test section, $34.3 \mathrm{~cm}$ (13.5 in.) across the flats. A photograph of the cryogenic tunnel taken during initial assembly is shown in figure 2. From the vantage shown in figure 2, the fan is positioned in the lower left-hand corner of the tunnel circuit and the flow is counterclockwise.



Figure 2.- Langley pilot transonic cryogenic tunnel during initial assembly.

The tunnel is constructed of aluminum alloy and is encased in thermal insulation consisting of about $12.7 \mathrm{~cm}$ ( 5 in.) of urethane foam covered with a fiberglass-reinforced epoxy vapor barrier. The fan is driven by a 2.2-MW (3000-hp) variable-frequency motor. The Mach number of the cryogenic tunnel can be varied from about 0.05 to 1.2 at stagnation pressures varying from about 1 to $5 \mathrm{~atm}\left(1 \mathrm{~atm}=101.3 \mathrm{kN} / \mathrm{m}^{2}\right)$. The tunnel temperatures can be reduced to about $77.6 \mathrm{~K}\left(-320^{\circ} \mathrm{F}\right)$ by spraying liquid nitrogen directly into 
the flow circuit at two points, located just before the upper left-hand and just after the lower left-hand turns shown in the photograph of figure 2 . Viewing ports, $3.5 \mathrm{~cm}$ (1.4 in.) in diameter, are provided for monitoring the test section and the nitrogen injection zones.

Since this tunnel was to be the first transonic cryogenic tunnel in existence and was in itself a proof-of-concept vehicle, a rather extensive amount of instrumentation was incorporated in the design. The installed instrumentation allows measurement to be made of about 100 static pressures, 4 stagnation pressures, 30 temperatures, noise and turbulence at several places in the tunnel, dewpoint and oxygen content of the test gas, liquid nitrogen flow rate, exhaust mass flow rate, drive-shaft torque, and fan speed. In addition to the "permanently" installed devices, the instrumentation included pressure and temperature rakes for evaluating vertical, lateral, and horizontal pressure and temperature distributions in the test section.

\section{Proof-of-Concept Studies}

Mach number calibration.- An extensive series of calibrations were conducted to determine the Mach number distribution in the test section. The operating envelope was covered in detail, with special attention given to obtaining comparisons which would indicate any disparities that might occur between results at ambient and at cryogenic temperatures. The calibrations were made with a 1.9 -cm-diameter (3/4-in.) pressure probe at the center line which surveyed about $102 \mathrm{~cm}$ (40 in.) of the test section. A photograph of the pressure probe installed in the test section is shown in figure 3. (In this photograph



Figure 3.- Pressure calibration probe installed in test section. 
and in several subsequent photographs, the insulated plenum chamber and several of the test-section side plates have been removed to expose the test section.)

Figure 4 presents a sample of results which were obtained for two different test conditions. The results shown in the upper portion of the figure indicate the local Mach number $M_{l}$ distribution in the test section at a stagnation pressure of $1.2 \mathrm{~atm}$ and a cryogenic temperature of about $116 \mathrm{~K}\left(-251^{\circ} \mathrm{F}\right)$. The Mach number distribution shown in the lower portion of the figure was recorded at a stagnation pressure of $5 \mathrm{~atm}$ and a temperature of $332 \mathrm{~K}\left(137^{\circ} \mathrm{F}\right)$. In both cases the flow similarity parameters were almost identical; the free-stream Mach number was 0.90 and the Reynolds number was about $64 \times 10^{6}$ per meter $\left(19.5 \times 10^{6}\right.$ per foot). In figure 4 , center of rotation (C.R.) stations are indicated for two different models. (The two-dimensional and three-dimensional model studies are discussed in subsequent sections of this paper.) For the indicated test region, the maximum "scatter" in the local Mach number was about 0.008 . Also, there is a marked similarity between the two calibrations, and in both cases there are about 51 to $76 \mathrm{~cm}$ (20 to $30 \mathrm{in.)}$ of "good" flow upstream of the model. These results are typical of the high subsonic calibrations which were obtained. The deviation in Mach number was considerably less at the lower Mach numbers but was increased at the higher Mach numbers, with shorter regions of "good" flow. It should be noted, however, that at this time no attempt has been made to optimize the test-section geometry to improve the flow characteristics at the higher Mach numbers.



Temperature-distribution calibrations.- As mentioned previously, the wide range of operating temperatures is obtained by spraying liquid nitrogen directly into the tunnel circuit to cool the structure and test gas and to remove the heat added to the stream by the drive fan. Because of this method of cooling, the uniformity of the temperature distribu- 
tion was one of the areas of concern at the beginning of the cryogenic studies at Langley. In order to determine the extent of the mixing process and to evaluate the temperature distributions in the circuit, a temperature survey ring was placed just upstream of the screens in the tunnel. A photograph of the survey ring is shown in figure 5.



Figure 5.- Temperature survey ring installed in screen section of tunnel.

The ring incorporated 24 thermocouples which were evenly spaced along 8 spokes $45^{\circ}$ apart. The screen section was located just downstream of the turn in the tunnel circuit shown at the upper right-hand portion of figure 2 .

Figure 6 indicates typical examples of the distributions which were determined with the temperature survey ring. These particular samples were taken at a test-section Mach number of 0.85 and a stagnation pressure of 5 atm. The results shown at the left were recorded when the tunnel stagnation temperature $\mathrm{T}_{\mathrm{t}}$ was $326 \mathrm{~K}\left(127.7^{\circ} \mathrm{F}\right)$ and the data at the right were taken at a cryogenic temperature of $104.6 \mathrm{~K}\left(-271.4^{\circ} \mathrm{F}\right)$. It should be mentioned that the tunnel stagnation temperature $\mathrm{T}_{\mathrm{t}}$ used in the reduction of data is measured with a refined, individual temperature sensor located just upstream of the temperature survey ring toward the turn vanes. (See small insert sketch in fig. 6.) The symbols included with the sketch of the survey ring indicate the locations of the various measurements shown in the temperature distribution plots. The temperature plots indicate the temperature variations across the calibration ring. In both cases, it was slightly warmer near the walls of the tunnel, with a standard deviation $\sigma$ (measure of dispersion around the mean) of only about $0.56 \mathrm{~K}\left(1^{\circ} \mathrm{F}\right)$ at both the cryogenic and ambient stagnation temperatures. 




Figure 6.- Temperature distributions in screen section. $M_{\infty}=0.85 ; p_{t}=5$ atm.

Since the survey ring was located upstream of the smoothing screens and contraction section (see sketch, fig. 6), it might be expected that a more uniform distribution would occur in the test section than at the survey ring. This did prove to be the case. Of ultimate concern, of course, is the temperature distribution of the test gas in the test section. A temperature survey rake was used which could be oriented across the test section in vertical, horizontal, or lateral positions. The photograph of figure 7 shows the rake mounted in the vertical position. The rake consisted of seven evenly spaced thermocouple probes which protruded from a streamlined strut support.



Figure 7.- Temperature calibration rake installed in test section. 
An example of test-section temperature distributions which were determined at Mach 0.85 at a near-maximum stagnation pressure and a cryogenic stagnation temperature is shown at the right of figure 8 . These results indicate the temperatures which were determined at the various probe positions. The results show the excellent distribution which was obtained at a cryogenic temperature of about $120 \mathrm{~K}\left(-244^{\circ} \mathrm{F}\right)$. (Standard deviation $\sigma$ was $0.28 \mathrm{~K}\left(0.5^{\circ} \mathrm{F}\right)$.) When test-section temperature distributions were taken at the very beginning of a series of tests, that is, just after tunnel "cool down," there was a moderate peak in the distributions, indicating slightly warmer temperatures near the testsection walls and plenum chamber. This trend disappeared rapidly but it was very similar to the behavior of the distributions determined with the survey ring in the screen section of the tunnel.
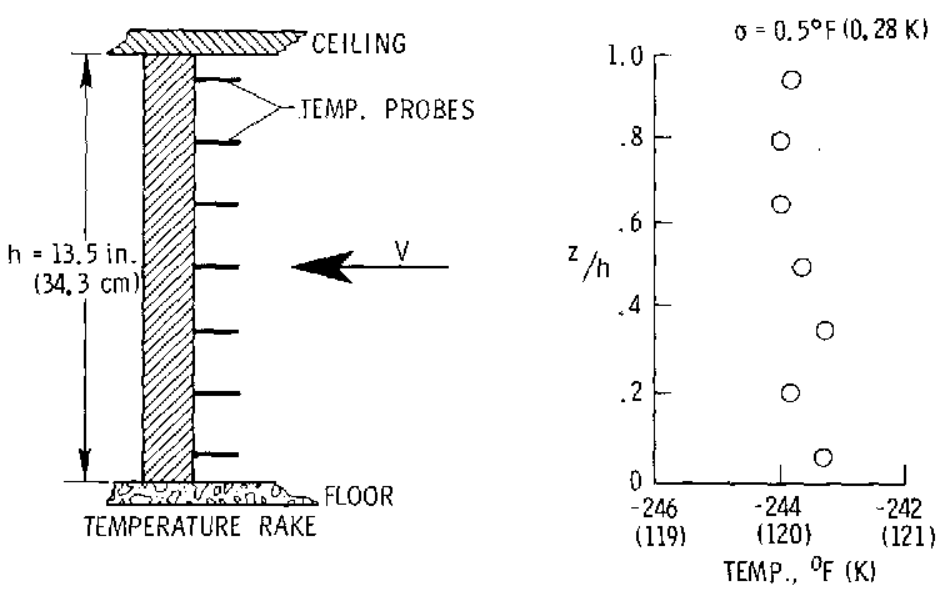

Figure 8. - Temperature distribution in test section. $M_{\infty}=0.85 ; p_{t}=5$ atra.

The temperature studies have shown remarkably good distributions. This is particularly obvious in view of the fact that it is not uncommon for conventional ambienttemperature wind tunnels to have temperature gradients of over $11 \mathrm{~K}\left(20^{\circ} \mathrm{F}\right)$ in the test section. These encouraging results indicate that cooling by injecting liquid nitrogen directly into the tunnel circuit is practical at the power levels required for transonic testing and that good temperature distribution can be obtained by using a simple liquid nitrogen injection system.

Noise level measurements. - In wind-tunnel testing, background noise in the test section is of concern since excessive noise levels can obstruct the proper simulation of unsteady aerodynamic parameters usually of interest in dynamic tests. In addition, testsection noise can affect angle-of-attack measurements, boundary-layer transition, and certain static or steady-state parameters. Because of the large reductions in drive power as temperature is reduced, it was expected that background noise would be reduced when a given Reynolds number was obtained at cryogenic temperatures rather than at ambient temperature. A preliminary study was made to check this hypothesis. 
The photograph shown in figure 7 shows cryogenic microphones, one mounted in the plenum area and the other mounted flush with the wall in the test section. The results shown in figure 9 indicate some trends that were determined with the test-section microphone. Sound pressure level $\mathrm{L}_{\mathrm{p}}$ in decibels is plotted against unit Reynolds number at



a Mach number of 0.80 for various pressure and temperature conditions. The noise levels are presented in terms of the broadband $(10 \mathrm{~Hz}$ to $20 \mathrm{kHz})$ sound pressure level, with the reference pressure taken to be $20 \mu \mathrm{N} / \mathrm{m}^{2}\left(2.9 \times 10^{-9} \mathrm{lbf} / \mathrm{in}^{2}\right)$. These measurements were made during the testing of a two-dimensional airfoil at an angle of attack of $3^{\circ}$, and the absolute level of the noise measurements may appear too high. The results, therefore, do not represent a pure indication of the minimum background noise and should only be used as comparative levels to indicate the general effects of changes in pressure and temperature. In figure 9, the tunnel conditions (stagnation pressure and temperature) at which the noise measurements were taken are shown adjacent to each of the plotted points. It will be noted that at a constant Reynolds number of about $62 \times 10^{6}$ per meter $\left(19 \times 10^{6}\right.$ per foot), operating at a temperature of $114 \mathrm{~K}\left(-254.5^{\circ} \mathrm{F}\right)$ allowed the desired Reynolds number to be obtained at the lower pressure of $1.17 \mathrm{~atm}$ and reduced the sound pressure level by about $10 \mathrm{~dB}$.

The results shown across the upper portion of figure 9 indicate that by reducing the tunnel temperature to $108 \mathrm{~K}\left(-265.3^{\circ} \mathrm{F}\right)$ at a constant pressure of about $4.9 \mathrm{~atm}$, the Reynolds number is increased by a factor of 4.6 with only a $1-\mathrm{dB}$ increase in noise level. It might be expected that a reduction in noise level would occur at the higher stagnation pressures when the tunnel was operated at cryogenic temperatures, because of reduced power requirements. (See fig. 1.) Although the slight increase in level is not completely 
understood at this time, it is believed to be associated with an increase in noise arising from the increased rate of nitrogen being exhausted from the tunnel at the cryogenic temperatures. If this is the case, special attention to the design of the nitrogen exhaust systems could very possibly result in additional noise reductions at all operating conditions.

An extensive analysis of test-section noise has not been made; however, these limited results have indicated some promising trends. It appears that with regard to tunnel noise level, the primary advantage of operating at cryogenic temperatures is the ability to obtain a given Reynolds number at a lower stagnation pressure and thus with a lower sound pressure level.

Experimental and theoretical drive-power and fan-speed studies.- During the initial calibrations and aerodynamic testing, measurements were made of both the drive-shaft torque and fan speed to permit comparisons with theoretical predictions at various conditions of temperature, pressure, and Mach number. At this time, the drive-power results have not been completely evaluated. However, from preliminary data based on power supplied to the drive motor, it appears that drive power varies roughly as predicted; that is, for constant pressure and Mach number, power varies directly with the speed of sound, or as $\sqrt{\mathrm{T}}$.

A sufficient number of fan-speed measurements have been made to evaluate the agreement between theory and experimental results. Figure 10 shows the theoretical and

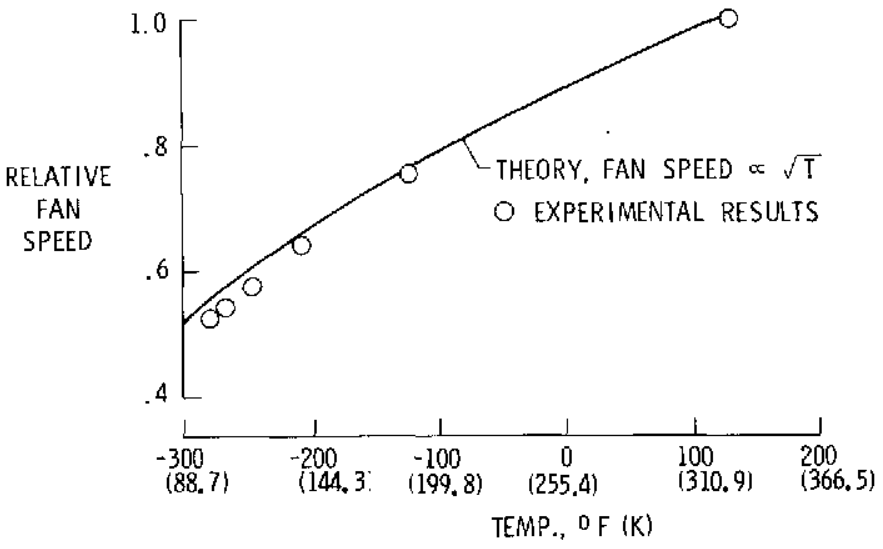

Figure 10.- Theoretical and experimental fan speeds at $M_{\infty}=0.85 . \quad p_{t} \approx 4.95$ atm.

experimental variation of fan speed with temperature at a Mach number of 0.85 and a constant stagnation pressure of about $4.95 \mathrm{~atm}$. The Reynolds numbers for this series of studies varied from about $62 \times 10^{6}$ per meter $\left(19 \times 10^{6}\right.$ per foot $)$ at the highest temperature to $328 \times 10^{6}$ per meter $\left(100 \times 10^{6}\right.$ per foot) at the lowest cryogenic temperature. The experimental results, shown by the circular symbols, indicate that the fan speed actually decreases somewhat faster than predicted by simple theory (speed $\propto \sqrt{T}$ ). Thus, 
at cryogenic temperatures the greatly increased Reynolds number has, as would be expected, a beneficial effect on tunnel performance and/or fan efficiency.

Two-dimensional airfoil study.- As noted in reference 3, the theoretical real-gas studies which have been made at the Langley Research Center indicate that for moderate operating pressures, the flow characteristics are insignificantly affected by the real-gas "imperfections" of nitrogen at cryogenic temperatures. Shortly after the initial tunnel calibrations, a series of two-dimensional airfoil tests were conducted to provide experimental confirmation of the cryogenic concept. The configuration selected for these studies was a 12-percent-thick, NACA 64-series airfoil equipped with pressure orifices. A photograph of the model is shown in figure 11 . The $13.7-\mathrm{cm}$-chord (5.4-in.) airfoil completely



Figure 11.- Proof-of-concept, two-dimensional airfoil model.

spanned the $34.3-\mathrm{cm}(13.5-i n$.$) test section. The sketch shown at the lower left of the$ figure indicates that at subcritical speeds, the 64-series airfoils have a "flattop" velocity distribution, similar to the upper surface distribution of current supercritical designs. A photograph of the model installed in the test section of the cryogenic pilot tunnel is shown in figure 12.

There were several conditions which were selected to assure a pure and adequate cryogenic evaluation: (1) Tests at ambient and cryogenic temperatures were to be made in the same tunnel, on the same model, at identical Mach numbers and Reynolds numbers; (2) the airfoil was to be tested with free transition to allow any possible temperature effect on boundary-layer development; (3) the symmetrical airfoil was to be tested at a lift coefficient of zero to eliminate any shape or angle-of-attack change due to dynamic-pressure differences; and (4) the test Mach number would exceed the leading-edge Mach number of typical sonic transport designs. 


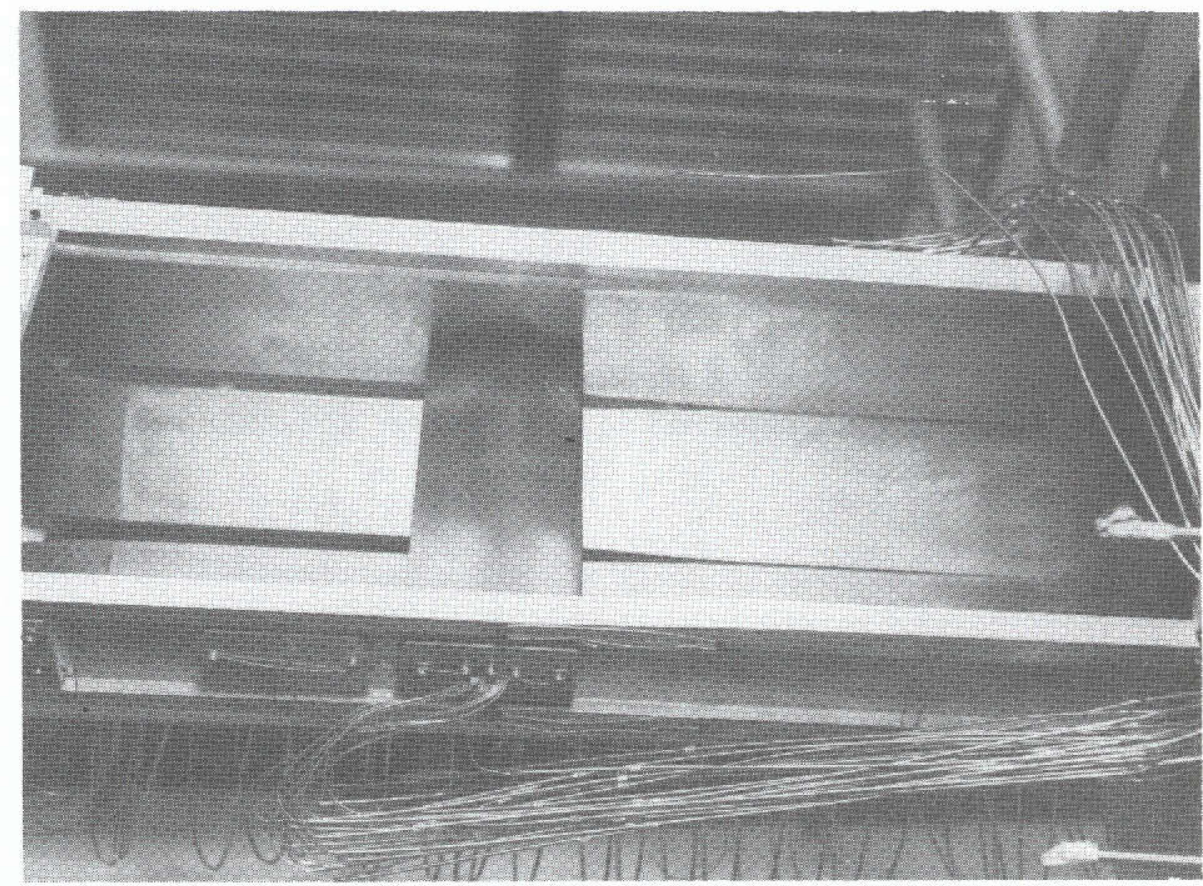

I-73-8915

Figure 12.- Two-dimensional airfoil installed in test section.

Figure 13 illustrates the operating envelope considered in the two-dimensional airfoil studies. Stagnation pressure $\mathrm{p}_{\mathrm{t}}$ is plotted against unit Reynolds number as well as Reynolds number based on model chord. The operating envelope is bounded by the stagnation pressure limits (horizontal lines) and stagnation temperature limits (diagonal curves). The curves for the theoretical saturation limits associated with the local and free-stream Mach numbers are indicated. The circular symbols illustrate the conditions

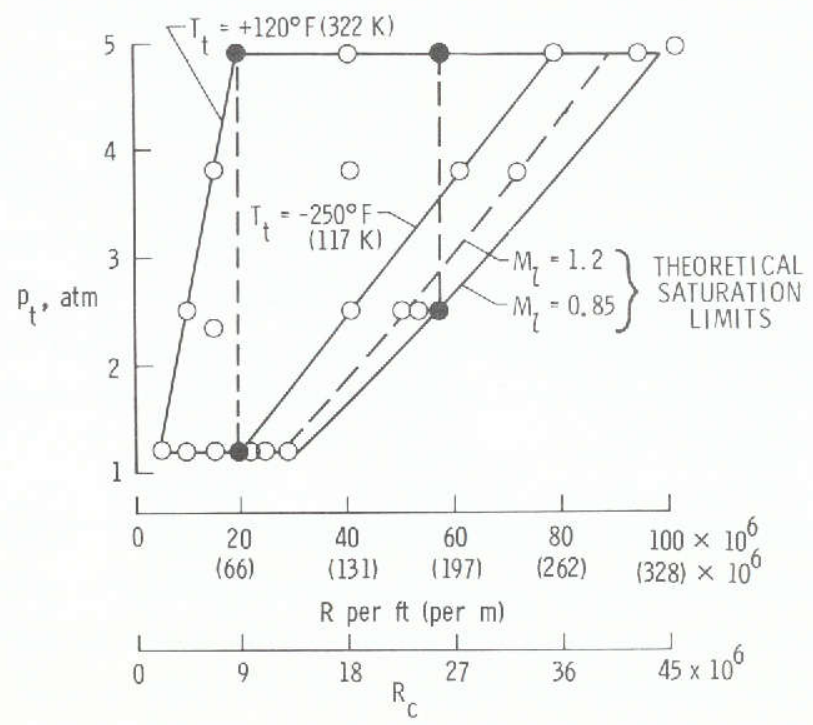

Figure 13.- Test conditions for two-dimensional airfoil at $M_{\infty}=0.85$. 
for tests performed at $M_{\infty}=0.85$ and Reynolds numbers ranging from about $14.8 \times 10^{6}$ to $328 \times 10^{6}$ per meter $\left(4.5 \times 10^{6}\right.$ to $100 \times 10^{6}$ per foot $)$. In the following paragraphs, pressure distributions that were obtained on the two-dimensional airfoil at $M_{\infty}=0.85$ will be discussed. The test conditions for these distributions are shown on this envelope as the solid symbols.

Figure 14 shows a comparison of the upper surface pressure distributions obtained at identical Reynolds numbers at both ambient and cryogenic temperatures for freestream Mach numbers of 0.75 and 0.85 . The angle of attack was $0^{\circ}$. The circular symbols denote pressure coefficients obtained at a stagnation pressure of about 4.9 atm and

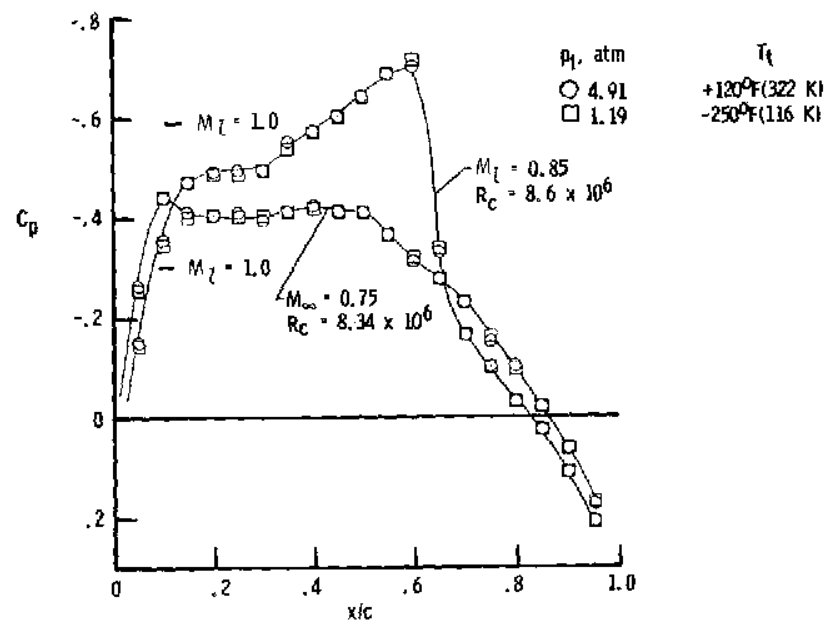

Figure 14.- Pressure distributions on two-dimensional airfoil at
ambient and cryogenic conditions. $\alpha=0^{\circ}$.

ambient temperature. The square symbols denote coefficients obtained at about $1.2 \mathrm{~atm}$ and a cryogenic temperature of $116 \mathrm{~K}\left(-250^{\circ} \mathrm{F}\right)$.

Several types of flow phenomena are illustrated in figure 14 . The results for $M_{\infty}=0.75$ are unmistakably subcritical. The results for $M_{\infty}=0.85$ indicate that the local Mach number becomes sonic (at $C_{p}=-0.3$ ) and continues to increase until a strong recompression shock is produced. In both cases, there is excellent agreement between the results for cryogenic and ambient temperatures. This agreement was achieved despite the sensitivity of the airfoil to changes in Mach number and the large variation in the speed of sound at the two temperatures. These results clearly demonstrate the ability to set the tunnel conditions accurately and substantiate the theoretical findings that cryogenic gaseous nitrogen is a valid test medium.

Figure 15 shows an example of the studies which were made to examine the effects of the theoretical saturation boundaries. The variations of pressure coefficients are shown for two different pressure and temperature conditions at a Reynolds number $\mathbf{R}_{\mathrm{C}}$ of $27 \times 10^{6}$. The circular symbols are the results obtained at $139 \mathrm{~K}\left(-210^{\circ} \mathrm{F}\right)$, well above either the local or free-stream saturation boundary. The square symbols are the results 




Figure 15.- Fressure distributions on two-dimensional airfoil indicating
effects of operating at the theoretical saturation limit. $M_{\infty}=0.85$;
$\alpha=0^{\circ} ; \quad R_{c}=27 \times 10^{6}$.

obtained at a stagnation temperature which resulted in the saturation boundary just being reached at free-stream conditions. The excellent agreement between the results suggests that additional Reynolds number capabilities can be expected from the cryogenic wind tunnel by testing at temperatures approaching the theoretical free-stream saturation boundary.

The two-dimensional airfoil tests substantiated that cryogenic test conditions can be set accurately and that gaseous nitrogen is a valid transonic test medium which allows the achievement of high Reynolds numbers without increases in aerodynamic loading. In addition, this particular series of studies indicated that substantial additional increases in Reynolds number capability may be realized by testing beyond the theoretical local saturation boundaries.

Three-dimensional model tests. - Three-dimensional model tests have been made in the transonic cryogenic tunnel on a delta-wing model with a sharp leading edge, an aspect ratio of 1.07 , and a sweep of $75^{\circ}$. The overall length of the model was $20.0 \mathrm{~cm}(7.87 \mathrm{in}$.) and the maximum span was $10.4 \mathrm{~cm}$ (4.1 in.). A photograph of the model installed in the tunnel is shown in figure 16.

The purposes of the three-dimensional model tests were (1) to investigate any possible effects of cryogenic conditions on the aerodynamic characteristics of a configuration having flow characterized by a separation-induced leading-edge vortex; and (2) to obtain cryogenic experience with an electrically heated strain-gage balance and the accompanying sting, a strut, and angle-of-attack measuring devices. (Similar tests with satisfactory results had been made previously in the low-speed cryogenic tunnel at Langley with a water-heated balance (ref. 2).) 




Figure 16.- Three-dimensional delta-wing model installed in test section.

The preliminary results of these current studies were encouraging. Some problems have been encountered with respect to balance zero shifts; however, the results obtained with the three-component balance show that angle-of-attack effects are duplicated at ambient and cryogenic temperatures and that electrically heated balances, as well as the previously studied water-heated balances, are feasible for cryogenic operation. In addition, there are no fundamental problems associated with measuring angle of attack at cryogenic temperatures, and the pitching-moment, axial-force, and normal-force results indicate that flows with leading-edge vortex effects are duplicated properly at cryogenic temperatures.

An example of the angle-of-attack effects are shown in figure 17, which shows the variation of normal-force coefficient with angle of attack at Mach numbers of 0.85 and 1.1. Although the primary purpose of this phase of the study was to obtain comparisons between results at ambient and cryogenic temperatures, theoretical predictions of normal force by 

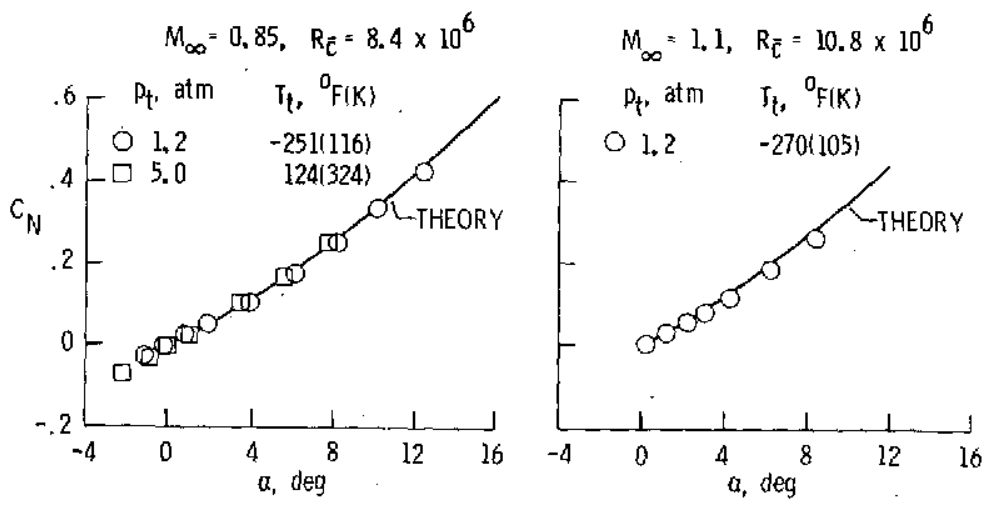

Figure 17.- Normal-force coefficients determined for three-dimensional model at ambient and cryogenic temperatures.

the method of reference 4 have been included with the experimental results. (See solid lines.) These predictions were included in this presentation to provide a basis for comparison at $M_{\infty}=1.1$, since at this Mach number, the increased aerodynamic loadings required to obtain experimental data at ambient temperature would have resulted in forces and moments exceeding the limits of the strain-gage balance system. At $\mathrm{M}_{\infty}=0.85$, the circular symbols indicate experimental results obtained at a stagnation pressure of $1.2 \mathrm{~atm}$ and a cryogenic temperature of about $116 \mathrm{~K}\left(-251^{\circ} \mathrm{F}\right)$. The results represented by the square symbols were taken at a stagnation pressure of $5 \mathrm{~atm}$ and a temperature of $324 \mathrm{~K}\left(124^{\circ} \mathrm{F}\right)$. The Reynolds number, based on model geometric chord, was $8.4 \times 10^{6}$ for both sets of data. As can be seen, there is good agreement between theory and the experimental results obtained at both ambient and cryogenic temperatures. At the right of the figure, the cryogenic results for $M_{\infty}=1.1$ are presented and compared with theory and appear to confirm also that there are no extraneous temperature effects. The small differences are not surprising, since experience has indicated that at supersonic speeds there is generally good agreement with theory at low angles of attack and that the theory predicts slightly more vortex lift than is actually produced at the higher angles of attack.

The three-dimensional model results have provided additional evidence that cryogenic nitrogen is a valid test gas even under conditions of separated and reattached (vortex) flow. In addition, there has been no indication of any major problem areas associated with obtaining angle-of-attack or strain-gage balance measurements at cryogenic temperatures.

\section{CONCLUSIONS}

The Langley pilot transonic cryogenic tunnel has recently been developed. Validation tests of this tunnel have led to the following conclusions: 
1. The cryogenic approach offers a feasible method for acquiring transonic test results at high Reynolds number with reduced levels of dynamic pressure and tunnel drive power.

2. With the unique ability to control tunnel temperature, pressure, and Mach number separately, it is possible to determine independently the effects of dynamic pressure (aeroelasticity), Reynolds number, and Mach number on the aerodynamic characteristics of the model under test.

3. Cooling with liquid nitrogen is practical at the power levels required for transonic testing. Test temperature is easily controlled and good temperature distribution obtained by using a simple nitrogen injection system.

4. Test-section noise level is reduced when a given Reynolds number is obtained by operating at cryogenic temperatures.

5. Two-dimensional and three-dimensional model tests have substantiated that cryogenic test conditions can be set accurately and that cryogenic gaseous nitrogen is a valid test medium.

6. Testing at temperatures several degrees beyond the local supersaturation boundary have been accomplished with no detectable effect on the pressure distribution over a two-dimensional airfoil.

Langley, Research Center,

National Aeronautics and Space Administration, Hampton, Va., January 6, 1975.

\section{REFERENCES}

1. Smelt, R.: Power Economy in High-Speed Wind Tunnels by Choice of Working Fluid and Temperature. Rep. No. Aero. 2081, Brit. R.A.E., Aug. 1945.

2. Goodyer, Michael J.; and Kilgore, Robert A.: High-Reynolds-Number Cryogenic Wind Tunnel. AIAA J., vol. 11, no. 5, May 1973, pp. 613-619.

3. Kilgore, Robert A.; Adcock, Jerry B.; and Ray, Edward J.: Flight Simulation Characteristics of the Langley High Reynolds Number Cryogenic Transonic Tunnel. ALAA Paper No. 74-80, Jan.-Feb. 1974.

4. Polhamus, Edward C.: Charts for Predicting the Subsonic Vortex-Lift Characteristics of Arrow, Delta, and Diamond Wings. NASA TN D-6243, 1971. 